\title{
A NOTE ON THE CONTINUITY OF LOCAL TIMES ${ }^{1}$
}

\author{
DONALD GEMAN
}

\begin{abstract}
Several conditions are given for a stochastic process $X(t)$ on $[0,1]$ to have a local time which is continuous in its time parameter (for example, in the Gaussian case, the integrability of $\left[E(X(t)-X(s))^{2}\right]^{-1 / 2}$ over the unit square). Furthermore, for any Borel function $F$ on $[0,1]$ with a continuous local time, the approximate limit of $|F(s)-F(t)| /|s-t|$ as $s$ $\rightarrow t$ is infinite for a.e. $t \in[0,1]$ and $\{s \mid F(s)=F(t)\}$ is uncountable for a.e. $t \in[0,1]$.
\end{abstract}

1. For a real, Borel function $F(t), 0 \leqslant t \leqslant 1$, put

$$
\mu_{t}(B)=m\left(F^{-1}(B) \cap[0, t]\right), \quad B \in \mathscr{B},
$$

where $m(d x)$ (or just $d x$ ) is Lebesgue measure on the real Borel $\sigma$-field $\mathscr{B}$. If $\mu_{1}$ is absolutely continuous with respect to $m$, we call $\alpha_{t}(x) \equiv d \mu_{t}(x) / d m$, $0 \leqslant t \leqslant 1,-\infty<x<\infty$, the local time of $F$. Local times are assumed to be chosen jointly measurable and nondecreasing, right-continuous in $t$ for every $x$; such versions always exist. The measure corresponding to $\alpha .(x)$ is then denoted $\alpha(d t, x)$ and represents the "time spent" by $F$ in the level $x$ during $d t$.

Let $X(t, w), 0 \leqslant t \leqslant 1$, be a separable and measurable stochastic process over a probability space $(\Omega, \mathscr{F}, P)$. In the Gaussian case, mean 0 , Berman [1] showed that if

$$
\int_{0}^{1} \int_{0}^{1} \frac{d s d t}{\left[E(X(s)-X(t))^{2}\right]^{1 / 2}}<\infty,
$$

then almost every sample path $X(\cdot, w)$ has a local time, say $\alpha_{t}(x, w)$, and then, in a series of papers, gave various additional conditions for a version of $\alpha_{t}(x, w)$ to be jointly continuous in $(t, x)$ a.s. (for example, in the stationary increments case, (1) with the exponent $1 / 2$ replaced by $1+\varepsilon$ for some $\varepsilon>0$; see [3]). Berman [3] also observed that for any Borel function $F$, the joint continuity of its local time implies

$$
\text { ap } \lim _{s \rightarrow t}\left|\frac{F(s)-F(t)}{s-t}\right|=+\infty
$$

for every $t \in(0,1)$ (where "ap lim" stands for approximate limit-see [6, p.

Received by the editors July 11, 1975.

AMS (MOS) subject classifications (1970). Primary 60G15, 60G17, 26 A24.

1 This work was partially supported by NSF Grant MPS72-04813 A03. 
220] and below), and in [2] proved that if (1) holds, and $X(\cdot, w)$ is continuous, then, with probability one, $\{s \in[0,1] \mid X(s, w)=X(t, w)\}$ is infinite for a.e. $t$ $\in[0,1]$.

In this note we will prove:

TheOREM A. Suppose $F$ has a local time $\alpha_{t}(x)$ which is continuous in $t$ for a.e. $x$. Then

(a) ap $\lim _{s \rightarrow t}|(F(s)-F(t)) /(s-t)|=+\infty$ for a.e. $t \in[0,1]$,

(b) $L_{t}=\{s \in[0,1] \mid F(s)=F(t)\}$ is uncountable for a.e. $t \in[0,1]$.

THEOREM B. Let $X(t, w)$ be a stochastic process for which either

$$
\int_{0}^{1} \sup _{\varepsilon>0} \frac{1}{\varepsilon} P(|X(s)-X(t)| \leqslant \varepsilon) d s<\infty \quad \text { for a.e. } t \in[0,1]
$$

or

(II) For each $0 \leqslant s \leqslant 1$, the distribution of $X(s)$ is absolutely continuous; for each $0 \leqslant s, t \leqslant 1$, the (joint) distribution of $(X(s), X(t))$ is absolutely continuous on an open strip $B$ (independent of $s, t$ ) containing the diagonal $x=y$, and the density $g_{s, t}(x, y)$ on $B$ is continuous on the diagonal and satisfies

$$
\int_{0}^{1} \int_{0}^{1} \sup _{x, y \in B} g_{s, t}(x, y) d s d t<\infty .
$$

Then, with probability one, $X(t, w)$ has a local time $\alpha_{t}(x, w)$ which is continuous in $t$ for a.e. $x$.

In the Gaussian case, (I) becomes

$$
\int_{0}^{1} \frac{d s}{\left[E(X(t)-X(s))^{2}\right]^{1 / 2}}<\infty \text { for a.e. } t \in[0,1],
$$

which is implied by (1). (However, the conclusions inferred from (1) can also be inferred from (4) by restricting $X(t, w)$ to a sequence $E_{n} \subset[0,1]$ with

$$
m\left([0,1] \backslash \bigcup_{n} E_{n}\right)=0
$$

and $\left[E(X(t)-X(s))^{2}\right]^{-1 / 2}$ integrable over each $E_{n} \times E_{n}$.) Condition (4) (or (1)), is weaker than the known sufficient conditions for the joint continuity of $\alpha$. Still in the Gaussian case (mean 0), (II) reduces to

$$
\int_{0}^{1} \int_{0}^{1} \frac{d s d t}{\left[\left(E X^{2}(t)\right)\left(E X^{2}(s)\right)-(E X(t) X(s))^{2}\right]^{1 / 2}}<\infty
$$

which was assumed in [5] for the existence of $\alpha$. For a continuous covariance, (5) implies (1), but not conversely, as seen by the process $W\left(t^{2}, w\right), W$ being ordinary Brownian motion. Nonetheless, if $X(t, w)$ satisfies (1), then the process $X(t, w)+\xi(w)$, where $\xi$ is standard normal and independent of $X$, has the same local time properties as $X$ and satisfies both (1) and (5). In the stationary case, (1), (4), and (5) are equivalent, and are implied by 


$$
\int_{0}^{1} \frac{d s}{\sigma(s)}<\infty, \quad \sigma^{2}(s)=E[X(s)-E(X(s) \mid X(u), u \leqslant 0)]^{2},
$$

which was assumed in [4] to get the continuity (in $t$ ) of $\alpha$.

The meaning of part (a) of Theorem $A$ is this: almost every $t \in(0,1)$ is a point of dispersion for the time spent by $F$ in every cone with vertex at $(t, F(t))$ and axis parallel to the abscissa. In particular, almost every $t \in(0,1)$ is an approximate "knot-point" (a term due to G. C. Young) of $F$, i.e. both approximate upper Dini derivates are $+\infty$ and both lower ones are $-\infty$ (and hence likewise for the ordinary derivates). (This follows from the DenjoyKhintchine theorem [6, p. 295].) It is noteworthy that for any Borel function $F$, the set of points $t$ at which $\lim _{s \rightarrow t}|F(s)-F(t)| /|t-s|=+\infty$ is of measure zero [6, p. 270]. This of course is false if lim is replaced by ap lim: "this rather unexpected fact was brought to light by V. Jarnik, who showed that there exist continuous functions $F$ for which the relation

$$
\text { ap } \lim _{h \rightarrow 0^{+}}|F(x+h)-F(x)| / h=+\infty
$$

holds at almost all points $x$ " [6, p. 297].

2. For brevity, the verification of the measurability of various sets and functions will be left aside.

Proof of Theorem A. (a) For each $0<t<1$ and $M>0$, set

$$
\begin{aligned}
D_{t, M} & =\{s \in[0,1]|| F(s)-F(t)|\leqslant M| t-s \mid\}, \quad \text { and } \\
\tau(t, M) & =\varlimsup_{\varepsilon \downarrow 0} \frac{1}{2 \varepsilon} m\left(D_{t, M} \cap(t-\varepsilon, t+\varepsilon)\right) .
\end{aligned}
$$

By definition, (2) holds at $t$ whenever $\tau(t, M)=0$ for every $M>0$.

It follows from the definition of $\alpha$ and a monotone class argument that

$$
\int_{0}^{1} f(s, F(s)) d s=\int_{-\infty}^{\infty} \int_{0}^{1} f(s, x) \alpha(d s, x) d x
$$

for every measurable $f \geqslant 0$. In particular, for every $0 \leqslant s<t \leqslant 1$ there is a full set $E_{s, t}$, i.e. $m\left(E_{s, t}^{c}\right)=0$, such that

$$
\lim _{\varepsilon \downarrow 0} \frac{1}{2 \varepsilon}\left(\mu_{t}[x-\varepsilon, x+\varepsilon]-\mu_{s}[x-\varepsilon, x+\varepsilon]\right)=\alpha((s, t), x) \text { for all } x \in E_{s, t} .
$$

Let $\mathcal{H}$ denote the collection of open intervals in $[0,1]$ with rational endpoints. Since $\mathcal{H}$ is countable,

$$
\lim _{\varepsilon \downarrow 0} \frac{1}{2 \varepsilon} \int_{B} I_{[0, \varepsilon]}(|F(s)-x|) d s=\alpha(B, x) \quad \forall B \in \mathcal{K},
$$

for all $x$ in a full set $E$. Now (7) remains valid with $x$ replaced by $F(t)$ for all $t$ in $G \equiv F^{-1}(E)$, which is full since $m\left(E^{c}\right)=0$ implies $m\left(F^{-1}\left(E^{c}\right)\right)=0$.

Now fix $B \in \mathcal{H}, t \in B \cap G$, and $M>0$. 


$$
\begin{aligned}
\alpha(B, F(t)) & \geqslant \varlimsup_{\varepsilon \downarrow 0} \frac{1}{2 \varepsilon} \int_{B \cap D_{t, M}} I_{[0, \varepsilon]}(|F(s)-F(t)|) d s \\
& \geqslant \varlimsup_{\varepsilon \downarrow 0} \frac{1}{2 \varepsilon} \int_{B \cap D_{t, M}} I_{[0, \varepsilon]}(M|t-s|) d s \\
& =\varlimsup_{\varepsilon \downarrow 0} \frac{1}{M}\left[\frac{1}{2 \varepsilon} m\left(B \cap D_{t, M} \cap(t-\varepsilon, t+\varepsilon)\right)\right] \\
& =\tau(t, M) / M .
\end{aligned}
$$

Letting $B \downarrow\{t\}$, we find that

$$
\sup _{M>0} \frac{\tau(t, M)}{M} \leqslant \alpha(\{t\}, F(t)), \quad t \in G
$$

where $\alpha(\{t\}, x)$ is the mass placed on $\{t\}$ by $\alpha(d s, x)$.

Finally, let $D=\{x: \alpha(\{s\}, x)=0 \forall s\}$ : by assumption, $m\left(D^{c}\right)=0$, and hence $m\left(F^{-1}\left(D^{c}\right)\right)=0$, i.e. $\alpha(\{s\}, F(t))=0 \forall s$ for a.e. $t \in[0,1]$. In particular, $\alpha(\{t\}, F(t))=0$ a.e.

(b) Having already noticed that the measure $\alpha(d s, F(t))$ is continuous for a.e. $t$, we need only check that (i) $\alpha_{1}(F(t))>0$ a.e. and (ii) $\alpha\left(L_{t}^{c}, F(t)\right)=0$ a.e.

As for (i), for any $B \in \mathscr{B}, B \subset[0,1]$,

$$
\int_{B} \alpha_{1}(F(s)) d s=\int_{-\infty}^{\infty} \alpha_{1}(x) \alpha(B, x) d x \geqslant \int_{-\infty}^{\infty} \alpha^{2}(B, x) d x,
$$

which is positive whenever $m(B)>0$. (The assumption in [2, Lemma 1.1] that $\alpha$ be square integrable is extraneous.) To obtain (ii), let $M_{x}=\{s \in[0,1] \mid$ $F(s)=x\}$ so that $L_{t}=M_{F(t)}$. From (6),

$$
0=\int_{0}^{1} I_{L_{f}^{c}}(t) d t=\int_{-\infty}^{\infty} \alpha\left(M_{x}^{c}, x\right) d x,
$$

in which case $\alpha\left(M_{x}^{c}, x\right)=0$ a.e., and in turn $\alpha\left(L_{t}^{c}, F(t)\right)=0$ for a.e. $t \in[0,1]$.

Proof of Theorem B. Assumption (I) implies

$$
\varliminf_{\varepsilon \downarrow 0} \frac{1}{2 \varepsilon} \int_{0}^{1} I_{[0, \varepsilon]}(|X(s)-X(t)|) d s<\infty \quad \text { for a.e. } t \in[0,1],
$$

for almost every $w \in \Omega$ (by taking the expected value of the random variable in (9) and using Fatou's lemma). However, for any Borel function $F(t)$ on $[0,1],(9)$-with $X$ replaced by $F$-is necessary and sufficient for a local time. To see this, let $G(x)=m\left(F^{-1}(-\infty, x] \cap[0,1]\right)$; it is a standard fact about the differentiation of measures that $G^{\prime}(x)$ exists, finite or infinite, for a.e. $x(d G)$, and that $G$ is absolutely continuous if and only if $G^{\prime}(x)<\infty$ a.e. $(d G)$. In other words,

$$
\lim _{\varepsilon \downarrow 0} \frac{1}{2 \varepsilon} \int_{0}^{1} I_{[0, \varepsilon]}(|F(s)-F(t)|) d s
$$

exists (possibly at $+\infty$ ) for a.e. $t \in[0,1]$, and $F$ has a local time if and only if 
the limit is actually finite for a.e. $t \in[0,1]$.

Next, proceeding as in the proof of Theorem A, we have

$$
\alpha(B, X(t, w), w)=\lim _{\varepsilon \downarrow 0} \frac{1}{2 \varepsilon} \int_{B} I_{[0, \varepsilon]}(|X(s, w)-X(t, w)|) d s, \quad \forall B \in \mathcal{K},
$$

for a.e. $t \in[0,1]$, all with probability one. Taking expected values and applying Fatou's lemma yields

$$
E \alpha(B, X(t)) \leqslant \int_{B} \sup _{\varepsilon>0} \frac{1}{\varepsilon} P(|X(t)-X(s)| \leqslant \varepsilon) d s, \quad \forall B \in \mathcal{H},
$$

for a.e. $t \in[0,1]$, and for such $t$ 's we can choose a sequence from $\mathcal{H}$ which decreases to $\{t\}$ and conclude that $E \alpha(\{t\}, X(t, w), w)=0$. Consequently with probability one,

$$
\begin{aligned}
0 & =\int_{0}^{1} \alpha(\{t\}, X(t, w), w) d t=\int_{-\infty}^{\infty} \int_{0}^{1} \alpha(\{t\}, x, w) \alpha(d t, x, w) d x \\
& =\int_{-\infty}^{\infty} \sum_{t \in[0,1]} \alpha^{2}(\{t\}, x, w) d x
\end{aligned}
$$

where the second equality uses (6).

Under condition (II), the existence of $\alpha$ results from an easy modification of the proof of Theorem 2 of [5]. As for continuity, let $\phi(\lambda ; x, w)$ be the Fourier transform of the (finite) measure $\alpha(d t, x, w)$ :

$$
\phi(\lambda ; x, w) \doteq \int e^{i \lambda t} \alpha(d t, x, w), \quad-\infty<\lambda, x<+\infty, \quad w \in \Omega .
$$

As is well known,

$$
\lim _{T \rightarrow \infty} \frac{1}{T} \int_{0}^{T}|\phi(\lambda ; x, w)|^{2} d \lambda=\sum_{t \in[0,1]} \alpha^{2}(\{t\}, x, w) .
$$

We will show that the expected value of the left-hand side of (10) is zero for a.e. $x$.

From (6), for almost every $w$,

$$
\int_{0}^{1} e^{i \lambda s} I_{[0, \varepsilon]}(X(s, w)-x) d s=\int_{x}^{x+\varepsilon} \phi(\lambda ; y, w) d y \quad \forall \lambda, x .
$$

Let $\varepsilon Z_{\varepsilon}(\lambda ; x, w)$ denote the left-hand side of (11). Almost surely, then, for every $\lambda$,

$$
\lim _{\varepsilon \downarrow 0} Z_{\varepsilon}(\lambda ; x, w)=\phi(\lambda ; x, w)
$$

for a.e. $x$. Using Fubini's theorem we obtain set $\Delta \in \mathscr{B}, m\left(\Delta^{c}\right)=0$, such that for every $x \in \Delta,(12)$ holds for $(m \times P)$-a.e. pair $(\lambda, w)$. For such $x$ 's, 


$$
\begin{aligned}
E \lim _{T \rightarrow \infty} \frac{1}{T} \int_{0}^{T}|\phi(\lambda ; x, w)|^{2} d \lambda & \leqslant \varliminf_{T \rightarrow \infty} E \frac{1}{T} \int_{0}^{T}|\phi(\lambda ; x, w)|^{2} d \lambda \\
& =\varliminf_{T \rightarrow \infty} \frac{1}{T} \int_{0}^{T} E|\phi(\lambda ; x, w)|^{2} d \lambda \\
& =\varliminf_{T \rightarrow \infty} \frac{1}{T} \int_{0}^{T} E \lim _{\varepsilon \downarrow 0}\left|Z_{\varepsilon}(\lambda ; x, w)\right|^{2} d \lambda \\
& \leqslant \underline{\lim _{T \rightarrow \infty}} \frac{1}{T} \int_{0}^{T} \underline{\lim _{\varepsilon \downarrow 0}} E\left|Z_{\varepsilon}(\lambda ; x, w)\right|^{2} d \lambda .
\end{aligned}
$$

Finally, by dominated convergence and the continuity of $g_{s, t}$ at $(x, x)$,

$$
\begin{aligned}
\varliminf_{\varepsilon \downarrow 0} E\left|Z_{\varepsilon}(\lambda ; x, w)\right|^{2} & =\varliminf_{\varepsilon \downarrow 0} \frac{1}{\varepsilon^{2}} \int_{0}^{1} \int_{0}^{1} \int_{x}^{x+\varepsilon} \int_{x}^{x+\varepsilon} e^{i \lambda(s-t)} g_{s, t}(u, v) d u d v d s d t \\
& =\int_{0}^{1} \int_{0}^{1} e^{i \lambda(s-t)} g_{s, t}(x, x) d s d t \\
& =\int_{0}^{1} e^{i \lambda s} G(s ; x) d s,
\end{aligned}
$$

where $G(s ; x)=\int_{0}^{1} I_{[0,1]}(s+t) g_{t, s+t}(x, x) d t$ is nonnegative and integrable $(d s)$ over $(-\infty, \infty)$. Hence

$$
\lim _{T \rightarrow \infty} \frac{1}{T} \int_{0}^{T} \int_{-\infty}^{\infty} e^{i \lambda s} G(s ; x) d s d \lambda=0 \text { for every } x,
$$

which concludes the proof.

REMARK. For a Gaussian process satisfying (1), we have $E \alpha(1, x)>0$ for a.e. $x$. Consequently, the proof of part (b) shows that, for almost every $x, \quad M_{x}(w)=\{t \in[0,1] \mid X(t, w)=x\}$ is uncountable with positive probability. Under further restrictions, Berman [3], Orey [5], and others have computed the a.s. Hausdorff dimension of $M_{x}(w)$.

\section{REFERENCES}

1. S. M. Berman, Local times and sample function properties of stationary Gaussian processes, Trans. Amer. Math. Soc. 137 (1969), 277-299. MR 39 \# 1009.

2. - Harmonic analysis of local times and sample functions of Gaussian processes, Trans. Amer. Math. Soc. 143 (1969), 269-281. MR 40 \#2155.

3. - Gaussian processes with stationary increments: Local times and sample function properties, Ann. Math. Statist. 41 (1970), 1260-1272. MR 42 \#6916.

4. D. Geman and J. Horowitz, Local times and supermartingales, Z. Wahrscheinlichkeitstheorie und Verw. Gebiete 29 (1974), 273-293. MR 50 \# 11476.

5. S. Orey, Gaussian sample functions and the Hausdorff dimension of level crossings, $\mathrm{Z}$. Wahrscheinlichkeitstheorie und Verw. Gebiete 17 (1971), 39-47.

6. S. Saks, Theory of the integral, Dover, New York, 1964. MR 29 \#850.

Department of Mathematics and Statistics, University of Massachusetts, Amherst, MASSACHUSETTS 01002 